\title{
Management of COVID-19 in patients after liver transplantation: Beijing working party for liver transplantation
}

\author{
Hongling Liu ${ }^{1} \cdot \mathrm{Xi} \mathrm{He}^{1} \cdot$ Yudong Wang ${ }^{2}$. Shuangnan Zhou ${ }^{1} \cdot$ Dali Zhang ${ }^{1}$. Jiye Zhu ${ }^{3} \cdot$ Qiang He $^{4}$ - Zhijun Zhu ${ }^{5}$. \\ Guangming $\mathrm{Li}^{6} \cdot$ Libo Sun $^{6}$ • Jianli Wang ${ }^{7}$. Gregory Cheng ${ }^{2} \cdot$ Zhenwen Liu $^{1} \cdot$ George Lau ${ }^{1,2}$
}

Received: 7 April 2020 / Accepted: 7 April 2020 / Published online: 10 April 2020

(c) Asian Pacific Association for the Study of the Liver 2020

\begin{abstract}
Annually, around 850 liver transplantation is performed in Beijing, China. Recently, the new coronavirus pneumonia (COVID-19) caused by 2019 novel coronavirus (2019-nCoV) has affected nearly 200 countries worldwide. 2019-nCov can cause severe lung disease, multiple-organ damage, and significant mortalities. Liver transplant recipients, because of long-term oral immunosuppressant effects, may be more susceptible to 2019-nCoV infection and have a worse prognosis than the general population. It is urgent to set up guidelines for the prevention, diagnosis, and treatment of COVID-19 in liver transplant recipients. In this article, we reviewed the clinical aspects of 2019-nCoV infection, characteristics of liver transplant recipients, immunosuppressant usage, and potential drug interactions to provide recommendations to clinical staff managing liver transplant recipients during the COVID-19 epidemic.
\end{abstract}

Keywords 2019-nCoV · COVID-19 · Liver transplantation · Management

\section{Introduction}

The pathogen causing a "mysterious" pneumonia in Wuhan, Hubei, since December 2019, has been identified as a novel coronavirus [1]. The World Health Organization (WHO) named this virus the 2019 new coronavirus (2019-nCoV) and the new coronavirus pneumonia as COVID-19. COVID19 has been included in the Class B infectious diseases stipulated in the Law of the People's Republic of China on the

Hongling Liu and Xi He contributed equally to this study.

Zhenwen Liu

liuzhenwen@medmail.com.cn

George Lau

gkklau@hnhmgl.com; gkklau@netvigator.com

1 Liver Transplantation Center, The Fifth Medical Center of Chinese PLA General Hospital, 100 West 4th Ring Middle Road, Beijing 100039, China

2 Humanity and Health Medical Group, Humanity and Health Clinical Trial Center, Unit 2101, 21F, No. 9 Queen's Road Central, Hong Kong, SAR, China

3 Department of Surgery, People's Hospital, Beijing Medical University, Beijing 100044, China
Prevention and Control of Infectious Diseases, and is managed as a Class A infectious disease [1].

The COVID-19 epidemic has spread rapidly in China and other countries worldwide. With effective measures, the number of new cases in China has decreased significantly. However, with the sharp increase in other countries, imported cases increase markedly in China.

Early diagnosis, early quarantine, and early treatment are important in COVID-19 prevention and control. Understanding its biological characteristics and clinical manifestations is crucial to set up proper guidelines [2]. This article summarized recent relevant publications and put forward

4 Department of Hepatobiliary and Pancreaticosplenic Surgery, Beijing Chaoyang Hospital, Capital Medical University, Beijing 100020, China

5 Clinical Center for Pediatric Liver Transplantation, Beijing Friendship Hospital, Capital Medical University, Beijing 100050, China

6 Department of General Surgery and Liver Transplant Center, Beijing You' an Hospital, Capital Medical University, Beijing 100069, China

7 Department of Liver Transplantation, The Third Medical Center of Chinese PLA General Hospital, Beijing 100039, China 
recommendations for management of liver transplantation during COVID-19 epidemic period.

\section{9-nCoV and its pathogenesis}

2019-nCoV is a single-stranded positive-strand RNA, betatype coronaviruses. It has four major structural proteins, namely fibrillin (S), envelope protein (M), small envelope protein $(\mathrm{E})$, and nuclear protein $(\mathrm{N})$. The initial attachment to the host cell is via binding of S protein to ACE2 receptor on host cell membrane [3].

2019-nCoV is sensitive to ultraviolet rays and heat. It can be inactivated by ether, $75 \%$ alcohol, $56{ }^{\circ} \mathrm{C}$ for $30 \mathrm{~min}$, and chlorine-containing disinfectant, chloroform [1]. The main transmission routes are droplet transmission and mucosal contact transmission [4]. There is also the possibility of aerosol transmission after exposure to high concentrations of aerosol in a relatively closed environment for long period. 2019-nCoV nucleic acid sequences can be detected in patients' eye secretions and feces, but whether transmission can occur remains to be confirmed [4]. 2019-nCoV infects all age groups, particularly the elderly and those with underlying diseases [4]. Organ transplant recipients are susceptible population.

Whether organ transplantation should be carried out during the COVID-19 epidemic remains controversial [1, 4]. Due to the unknown risks, some experts suggested that transplantation should be suspended. Alternatively, the recently released guidance [4] suggested that transplantation surgery could be carried out after careful risk assessments.

\section{Clinical characteristics of COVID-19 in liver transplant recipients}

Currently, the number of confirmed COVID-19 cases after liver transplantation is limited. In general, the clinical manifestations of COVID-19 were similar to general population.

\section{Incubation period}

The incubation period of 2019-nCoV infection is 1-14 days, mostly 3 to 7 days. Transmission can occur during the incubation period [1]. There is no evidence that the incubation period in liver transplant recipients is different.

\section{Clinical manifestations}

Fever

Fever is the first symptom of 2019-nCoV infection in most patients. However, in organ transplant recipients, there may be only low-grade fever or no fever at all [2]. Therefore, transplant physicians cannot relax their vigilance in afebrile patients $[1,2]$.

\section{Dry cough}

Dry cough is the main clinical manifestation of COVID-19 in the general population and transplant recipients $[1,5]$.

\section{Loss of smell and taste, and other symptoms}

Loss of smell and taste has been observed in many COVID19 patients.

Due to the immuno-suppressive state, COVID-19 may progress rapidly to acute ARDS in transplant recipients [1, 5].

Other common symptoms include fatigue, anorexia, nausea, nasal congestion, sore throat, myalgia, and diarrhea.

\section{Imaging findings}

The imaging findings of COVID-19 have common features with other viral pneumonia. Multiple small patchy shadows and interstitial changes with prominent extrapulmonary bands appear in early phase. Multiple ground-glass shadows, infiltrates, and lung consolidation occur during the progress phase. Pleural effusion is rare. Chest CT is the recommended imaging procedure. Compared with the general population, liver transplant recipients have more extensive lung lesions, multiple lesions, and more lower lobes involvement $[1,6]$.

\section{Laboratory tests}

Leucocyte count is normal or low. Lymphopenia is common. Some patients have elevated liver enzymes, muscle enzymes, myoglobin, and lactate dehydrogenase. Most have elevated C-reactive protein and erythrocyte sedimentation rate. Severe and critically ill patients are more likely to have elevated D-dimers, progressive lymphopenia, and increase in inflammatory markers $[1,5]$.

\section{Diagnosis of COVID-19 in liver transplant recipients}

\section{Suspected case}

A suspected case is a subject with epidemiological history mentioned in Sect. 3.1.1 and any two of the clinical manifestation criteria mentioned in Sect. 3.1.2. If there is no clear epidemiological history, the subject must have all three clinical manifestations [1]. 


\section{Epidemiological history}

In the 14 days before illness onset

1. History of residence in or entry and exit from a country with documented COVID-19 cases or

2. History of residence or visits to Wuhan and surrounding areas or

3. History of contact with COVID-19 patients or

4. Contact with patients with fever or respiratory symptoms from countries with case reports of COVID-19 or

5. Contact with patients with fever or respiratory symptoms from Wuhan and surrounding or

6. Residence or work in areas of suspected COVID-19 outbreaks (for example, two or more cases in the same building, school, or office).

\section{Clinical manifestation}

1. Fever or respiratory symptoms.

2. Normal or decreased leucocyte counts: lymphopenia.

3. One or more of the imaging characteristics described in Sect. 2.3.

\section{Confirmation of suspected case}

\section{COVID-19 is confirmed if}

1. Real-time fluorescent RT-PCR detection of 2019-nCoV nucleic acid is positive or

2. Gene sequences from patient's specimen and 2019$\mathrm{nCoV}$ are highly homologous or

3. Serum 2019-nCoV-specific IgM antibodies and IgG antibodies are positive ( $\mathrm{IgG}$ seroconversion or there is a fourfold increase from baseline).

COVID-19 is excluded if two consecutive tests of 2019$\mathrm{nCoV}$ nucleic acid (sampling time at least $24 \mathrm{~h}$ apart) are negative, and 2019-nCoV-specific $\mathrm{IgM} / \mathrm{IgG}$ antibodies are still negative after 7 days of onset.

2019-nCoV nucleic acid detection is performed in nasopharyngeal swabs, sputum, other lower respiratory tract secretions, blood, stool, and urine. Lower respiratory tract specimens (sputum or airway extracts) are preferred. Specimens should be submitted to testing centers as soon as possible after collection to maximize detection rates [1].

\section{Differential diagnosis}

Infections with influenza virus, adenovirus, respiratory syncytial virus, cytomegalovirus, Mycoplasma pneumoniae, Pneumocystis carinii, and other pathogens must be ruled out.

2019-nCoV testing is recommended in symptomatic liver transplant recipient with COVID-19 epidemiological history, even if the tests for common respiratory pathogens are positive $[1,2]$.

Liver dysfunction is observed in up to 50\% of COVID-19 patients $[5,7]$, mainly hepatocellular pattern, usually mild and resolves in 2-3 weeks. If a liver transplant recipient develops cholestatic liver injury or liver function deteriorates rapidly, other etiologies must be excluded before attributing to COVID-19.

\section{Management of liver transplant recipients during 2019-nCoV epidemics}

\section{Prevention of CoVID-19}

\section{Travel advice}

1. Liver transplant recipients and their family members in the same household should abide by the travel advisories issued by their government agencies and cancel unnecessary trips. Avoid travel on cruise ships and to places with 2019-nCoV outbreaks.

2. All patients who have returned from countries with COVID-19 should contact the transplant centers by phone or electronic messages regarding follow-up arrangements.

\section{Hospital visits}

Telemedicine is preferred over face-to-face consultations to avoid cross-infection during hospital visits.

Recommendations:

1. Reduce the frequency of examinations and imaging as much as possible.

2. If blood tests or imaging is required, perform them at a low-risk hospital or have blood samples taken at lowrisk centers and then deliver to the relevant hospital.

3. Telemedicine if possible.

\section{Personal hygiene and lifestyle}

1. Regular exercise at home: regular meals, avoid cold food, raw seafood and wild animals; regular sleep schedule.

2. Pay attention to hand hygiene; wash hands frequently; wear a mask when going out; avoid contact with patients 
with cough, fever, or infectious diseases; keep social distancing; regularly disinfect the environment.

3. Check body temperature daily.

4. Contact transplant centers promptly if develop fever and respiratory symptoms.

\section{Transplant center setup}

Recipients should be placed in single isolation rooms with bathroom facilities.

Close liaison with auxiliary departments such as radiology department so that the liver transplant recipients are separated in time and space from other patients.

\section{Transplant team}

Reviewing the reports published on SARS, MERS, and COVID-19, no mention is made on immunosuppression as a risk factor for mortality, and no fatality is reported to be linked to transplantation or other conditions requiring immunosuppressive treatment. Risk factors for poor outcome include advanced age, male sex, and presence of comorbidities [8-10].

Recommendations:

1. Treat any liver transplant candidate or recipient presenting with epidemiology history or clinical manifestations mentioned in Sect. 3.1 as suspected case.

2. Wear complete personal protective equipment during patient's care until 2019-nCoV infection is excluded.

3. Should self-quarantine after unprotected contact with a confirmed or suspected case until COVID-19 is excluded.

\section{Evaluation of potential liver transplant recipients and patients on transplant waiting list}

Currently, there is limited information on the impact of 2019-nCoV on patients with chronic hepatitis B infection, chronic liver disease, with decompensated liver cirrhosis or transplant candidates on waiting list.

Recommendations:

1. Follow measures mentioned in Sect. 4.1 to reduce the risk of 2019-nCoV infection.

2. Prescribe enough basic medicines such as antiviral drugs for $2-3$ months to reduce visits.

3. Avoid consulting potential liver transplant recipients or donors at liver clinic if they have fever and respiratory symptoms.

4. Living donation should not be performed on either a donor or recipient who has returned from countries with COVID-19 outbreaks or who have been exposed to con- firmed or suspected COVID-19 within 14 days. Donors should not be considered if they have fever and/or respiratory symptoms unless $2019-\mathrm{nCoV}$ is excluded.

\section{Management of COVID-19 liver transplant recipients}

Currently, it is unclear whether transplant recipients are more likely to develop more severe COVID-19. Only one case of fatal SARS [11] in liver transplant recipient is reported and none with MERS. As for 2019-nCoV, an experience in Bergamo shows that three of two hundred transplant recipients were tested positive for 2019-nCoV [10]. It is well known that immunocompromised people were susceptible to viral infection. However, immunosuppressed patients $>60$ years of age are at higher risk of infection with 2019-nCoV, and may have prolonged virus clearance $[1,5]$.

Recommendations:

1. Follow measures mentioned in Sect. 4.1 to reduce risk of 2019-nCoV infection.

2. No need to adjust the dose of immunosuppressive drugs.

3. Emphasize preventive measures mentioned in Sect. 4.1.

4. Transplant patients with fever and/or respiratory symptoms should inform the transplant centers promptly and avoid unscheduled visit to the clinic avoid inadvertent exposures.

\section{Immunosuppressive regimens in liver transplant recipients with COVID-19}

Currently, the impact of immunosuppressant on COVID-19 is unclear, and the recommendations for SARS/MERS are not validated because of short epidemic durations with limited cases. The potential role of corticosteroids in preventing mild COVID-19 from developing into severe pneumonia is controversial. WHO recommends avoiding the use of corticosteroids while treatment protocols in China recommend use for a short period of time in patients with rapid disease progression and evidence of marked inflammations. Also, there is concern that reducing or discontinuing immunosuppressant may cause acute graft rejection $[1,2]$. EASLESCMID position paper advises against immunosuppressive therapy reduction [12].

Recommendations:

1. No need to adjust the dose of immunosuppressive drugs in transplant recipients without COVID-19.

2. For patients with mild to moderate COVID-19, keep current immunosuppressant dosage and monitor patient's condition closely. Avoid prescription of medications that cause large fluctuations in tacrolimus plasma concentrations. 
3. For patients with severe or rapidly progressing COVID19 , reduce the amount of calcineurin inhibitor and consider stopping anti-metabolic drugs.

4. If decided to reduce the dose of steroids, keep at least a daily dose of $10 \mathrm{mg}$ prednisone or equivalence to avoid adrenal insufficiency.

5. Corticosteroids or other immunosuppressive therapies can be re-initiated with caution when the potential benefits outweighing their risks of discontinuation.

\section{COVID-19 therapy and potential drug interactions in liver transplant recipients}

The results of randomized controlled trials showed that lopinavir/ritonavir did not show clinical benefit in patients with severe COVID-19, and in some patients, treatment were discontinued early due to adverse drug reactions [13]. Lopinavir/ritonavir is a potent inhibitor of CYP3A4 and tacrolimus dose should be significantly reduced if lopinavir/ ritonavir is used. Animal studies have shown that ACEI and ARB can prevent severe pulmonary complications caused by COVID-19, and no data are available in humans. The European College of Cardiology Hypertension Committee recommends that patients with COVID-19 should continue to receive conventional antihypertensive treatment, including ACEI and ARB drugs.

Recommendations:

1. The current evidence does not support the use of lopinavir/ritonavir in liver transplant recipients with COVID19.

2. Continue the use of ACEI and ARB.

3. Use of traditional Chinese medicine therapy without evidence-based data is not recommended.

4. Do not stop or adjust the immunosuppressive regimen without consulting the doctors.

5. Avoid medicines that have an impact on immunosuppressive drug concentrations.

Acknowledgements We thank the Beijing Funding Project (Z161100000116058) and 302 Military Hospital Project (YNKT 2014006) for support.

\section{Compliance with ethical standards}

Conflict of interest Hongling Liu, Xi He, Yudong Wang, Shuangnan Zhou, Dali Zhang, Jiye Zhu, Qiang He, Zhijun Zhu, Guangming Li, Libo Sun, Jianli Wang, Gregory Cheng, Zhenwen Liu, George Lau declare that they do not have any conflict of interest.

\section{References}

1. China National Health Commission. National Health and Health Commission's plan for the diagnosis and treatment of new coronavirus pneumonia (trial version 7)

2. Chen S, Zhang WJ, Chen G, et al. Guidance on clinical diagnosis, treatment and transplantation of 2019-nCoV infected organ transplant recipients (First Edition). https://doi.org/10.3760/ cma.j.issn.0254. http://rs.yigle.com/m/yufabiao/1181145.htm

3. Liu Z, Xiao X, Wei X, et al. Composition and divergence of coronavirus spike proteins and host ACE2 receptors predict potential intermediate hosts of SARS-CoV-2. J Med Virol 2020. https://doi. org/10.1002/jmv.25726

4. Expert group on prevention and control of new coronavirus pneumonia of Chinese Preventive Medicine Association. New understanding of epidemiological characteristics of COVID-19. Chin J Epidemiol 2020;41(2):139-144

5. Guan WJ, Ni ZY, Hu Y, et al. Clinical characteristics of 2019 novel coronavirus infection in China. New Engl J Med 2020. https ://doi.org/10.1056/NEJMoa2002032

6. Shi HS, Han XY, Fan YQ, et al. Clinical and imaging features of pneumonia infected by 2019-nCoV. J Clin Radiol 2020. https:// doi.org/10.13437/j.cnki.jcr.20200206.002

7. Wang D, Hu B, Hu C, et al. Clinical characteristics of 138 hospitalized patients with 2019 novel coronavirus-infected pneumonia in Wuhan, China. JAMA. 2020 https://www.ncbi.nlm.nih.gov/ pubmed/32031570. Accessed $20 \mathrm{Feb} 2020$

8. Hui DS, Azhar EI, Kim YJ, et al. Middle East respiratory syndrome coronavirus: risk factors and determinants of primary, household, and nosocomial transmission. Lancet Infect Dis 2018;18:e217-e227

9. Chui AK, Rao AR, Chan HL, et al. Impact of severe acute respiratory syndrome on liver transplantation service. Transplant Proc 2004;36:2302-2303

10. D'Antiga L. Coronaviruses and immunosuppressed patients. The facts during the third epidemic. Liver Transpl 2020. https://doi. org/10.1002/lt.25756 (Epub ahead of print)

11. Kumar D, Tellier R, Draker R, et al. Severe acute respiratory syndrome (SARS) in a liver transplant recipient and guidelines for donor SARS screening. Am J Transplant 2003;3(8):977-981

12. Boettler T, Newsome PN, Mondelli MU, et al. Care of patients with liver disease during COVID-19 pandemic: EASL-ESCMID position paper. JHEP Rep. 2020 https://doi.org/10.1016/j.jhepr .2020 .100113

13. Cao B, Wang Y, Wen D, et al. A trial of lopinavir-ritonavir in adults hospitalized with severe Covid-19. N Engl J Med 2020. https://doi.org/10.1056/NEJMoa2001282

Publisher's Note Springer Nature remains neutral with regard to jurisdictional claims in published maps and institutional affiliations. 